\title{
Autonomía y juventudes en el Caribe sur nicaragüense
}

\section{Francisco Sequeira Rankin Consultor}

El proceso de autonomía del Caribe nicaragüense ha cumplido veintiséis años desde la aprobación de la Ley 28: Estatuto de la Autonomía de las Regiones de la Costa Atlántica de Nicaragua. La juventud de la costa caribe de la Nicaragua de hoy es la primera generación que ha crecido bajo el régimen autonómico. Al mismo tiempo, este grupo asume un peso cada vez mayor en la distribución demográfica de la región.

En el marco del programa Educación para el Éxito (EduÉxito), que ejecuta la Fundación para la Autonomía y el Desarrollo de la Costa Atlántica de Nicaragua (FADCANIC), se realizó una Encuesta de Conocimientos, Aspiraciones, Percepciones y Participación (ECAPP) de las juventudes en el régimen autonómico, titulada: La vigencia de la autonomía en la costa Caribe Sur de Nicaragua.

Este estudio fue realizado en cinco municipios de la Región Autónoma Atlántico Sur (RAAS): Desembocadura del Río Grande, Laguna de Perlas, Kukra Hill, Corn Island y Bluefields. La investigación tenía como objetivo analizar las actitudes y valoraciones de la juventud, en los diferentes grupos étnicos que pueblan la región, hacia el régimen autonómico como sistema político y expresión normada de democracia.

Para la realización del estudio fueron encuestados 726 jóvenes y adolescentes entre los dieciséis y veintinueve años, pertenecientes a los siguientes grupos étnicos: rama, garífuna, mayangna, miskito, ulwa, creole (kriol) y mestizo. Los temas de análisis fueron: aspiraciones y agencia de las juventudes, entendido como la capacidad de las personas para plantear y alcanzar sus metas; conocimientos y percepciones sobre el régimen autonómico; igualdad ante la ley; evaluación sobre el desempeño de las instituciones; valores, concepciones y expectativas sobre democracia, autonomía, política y participación. El estudio finaliza con un análisis acerca de cómo potenciar las oportunidades a través de programas con adolescentes y jóvenes.
En el presente resumen fueron analizados los resultados del estudio ECAPP a través de las interrelaciones entre autonomía y juventudes, con transición demográfica, identidades, régimen autonómico, agencia, participación y salud sexual y reproductiva - También se subraya la importancia de la población joven, con sus múltiples juventudes, para el desarrollo de la autonomía, en particular, por la transición demográfica que está aumentando la proporción relativa de jóvenes en la población. Igualmente se analiza el conocimiento y las percepciones de la juventud en torno a las instancias $\mathrm{y}$ actores del régimen autonómico establecidos en el marco normativo. Así mismo se revisa el concepto de agencia con referencia al sistema político de la autonomía y sus relaciones con la participación de la población joven en las cosas públicas, su acceso a la educación, el empleo, la recreación, la salud sexual y reproductiva y, más generalmente, en la obtención de su propio desarrollo integral. Finalmente se resume recomendaciones planteadas en el estudio, para futuras intervenciones con adolescentes y jóvenes de la costa caribe sur nicaragüense.

\section{Transiciones e identidades de juventudes}

En este momento, la costa caribe de Nicaragua, al igual que el resto del país, vive una transición demográfica transcendental en relación a su población joven, conocida como bono demográfico: la alta tasa de fecundidad y la reducción en la mortalidad infantil tienden a maximizar a cierto punto el número de personas potencialmente productivas en relación a las personas dependientes. Esta transición demográfica, que concluirá con el envejecimiento proporcional de la población debido al aumento de la esperanza de vida y la disminución de la tasa de fecundidad presenta, en los momentos y lugares donde ocurre, una oportunidad única para el desarrollo económico. 




Becarios y becarias de EduÉxito en Bluefields.

Para poder aprovechar esta oportunidad es necesario asegurar a los jóvenes el acceso a una educación de calidad, generadora tanto de actitudes y competencias personales y sociales, como de los conocimientos generales, técnicos y científicos pertinentes para las oportunidades de empleo que habrá que multiplicar en la región. Se necesitará para eso una planificación y ordenamiento territorial adecuados. Bajo estas condiciones, la región puede dar un salto cualitativo hacia adelante en términos de bienestar económico, reducción de las inequidades y el bienestar de las personas y la calidad de vida (Delgadillo/ UNFPA/ CELADE, 2010).

Esta evolución demográfica y potenciales desarrollos tienen también como consecuencia que la población joven tendrá una influencia predominante en la esfera política, por su peso porcentual entre los electores en tiempos de elecciones y, más generalmente, debido a su inserción en los diferentes mecanismos de participación. Así, las percepciones de estos adolescentes y jóvenes sobre el régimen autonómico del Caribe nicaragüense son vitales para el desempeño del mismo sistema.
La adolescencia es una etapa de vida en que se presentan cambios significativos biológicos y psicosociales. Además, durante la adolescencia se comienza a asumir los roles de las personas adultas, como trabajar y formar una familia. Sin embargo, los límites y roles de la adolescencia, como de la juventud, dependen en gran manera de la cultura en que se encuentren, así como de las condicionantes sociales y económicas.

Aunque el estudio no entra en la indagación de cómo los diferentes grupos étnicos construyen el imaginario de sus juventudes, las personas encuestadas plantearon en su mayoría que la juventud es una etapa de preparación para la vida adulta, mientras que una minoría planteó que es una fase para la diversión y felicidad.

Debido a que su construcción de juventudes es principalmente como un tiempo de preparación, ésta se refleja en las oportunidades que visualizan, que son de estudiar y trabajar. Dado que jóvenes y adolescentes participantes en el estudio son pobres en su mayoría, con $83.6 \%$ en situación de pobreza extrema, de acuerdo a las necesidades básicas insatisfechas, los obstáculos que identifican tienen que ver principalmente con la carencia de recursos, la falta de oportunidades de empleo y la pobreza. 


\section{Autonomía}

El estudio presenta resultados sobre el conocimiento y percepciones de la juventud, en torno a instituciones, actores, derechos y reglas del régimen autonómico, fundamentados en un marco normativo nacional e internacional.

A nivel internacional, tanto el Pacto International de Derechos Económicos, Sociales y Culturales y el Pacto Internacional de Derechos Civiles y Politicos, ambas de las Naciones Unidas, establecen, en su artículo 1, que:

"Todos los pueblos tienen el derecho de libre determinación. En virtud de este derecho establecen libremente su condición política y proveen asi mismo a su desarrollo económico, social y cultural."

Con mayor especificidad para los pueblos indígenas, la Declaración de las Naciones Unidas sobre derechos de los Pueblos Indigenas, en su artículo 4 plantea que:

"los pueblos indígenas, en ejercicio de su derecho a la libre determinación tienen derecho a la autonomía o al autogobierno en las cuestiones relacionadas con sus asuntos internos y locales, así como a disponer de medios para financiar sus funciones autónomas."

A nivel nacional, la Constitución Política de la República de Nicaragua reconoce el proceso de Autonomía de la Costa Atlántica de Nicaragua. El artículo 5 estipula la existencia del régimen de autonomía, y el Capítulo VI establece los derechos de las comunidades de las regiones autónomas. Esto se concretiza en el Estatuto de la Autonomía de la Regiones de la Costa Atlántica de Nicaragua, aprobado por la Asamblea Nacional en octubre de 1987, que establece las formas de administración autónoma: consejos y gobiernos regionales. La reglamentación del Estatuto fue aprobada en 2003, delimitando las funciones de las instituciones del régimen autónomo y los alcances de la autonomía. La aprobación, en 1993, de la Ley No. 162: Ley de Lenguas, así como la aprobación posterior de d la Ley No. 445: Ley del Régimen de Propiedad Comunal de los Pueblos Indígenas y Comunidades Étnicas de las Regiones Autónomas de la Costa Atlántica de Nicaragua y de los Ríos Bocay, Coco, Indio y Maíz contribuyeron a normar el funcionamiento de la autonomía de forma más precisa y operacional.
En términos de conocimiento sobre el régimen autonómico, el estudio muestra que aunque la mayoría de jóvenes encuestados tiene algún conocimiento sobre la autonomía, el $17 \%$ dijo no haber siquiera escuchado del mismo. En relación a los aspectos institucionales del régimen autonómico - es decir, sus actores, instancias, funcionamiento, derechos y normas - , la mitad de los encuestados manifestó desconocimiento.

Es importante reconocer que el hecho que la autonomía sea relativamente joven y todavía esté en proceso de definirse y consolidarse -razón por la cual no todas sus características actuales pueden ser parte de los programas escolares anteriormente vigentes-, explica en parte los bajos niveles de conocimiento que tiene, una parte substancial de la población joven, sobre la institucionalidad del régimen autonómico. Lógicamente, este desconocimiento relativo debe tener una importante influencia en las percepciones, actitudes y posibilidades de participar activamente en los procesos autonómicos, lo cual se refleja en la encuesta.

En el Informe de Desarrollo Humano de la Costa Caribe de Nicaragua 2005, se plantea que la Autonomía tiene dos dimensiones principales, la primera relacionada a la cultura e identidad, y la segunda a la autodeterminación política de las comunidades de forma democrática (PNUD, 2005: 18). Agrega que la autonomía es una condición esencial para el desarrollo humano y que requiere una ciudadanía activa basada en identidades y capitales sociales fuertes, una capacidad productiva dinámica y sostenible, y las instituciones y relaciones institucionales del buen gobierno (PNUD, 2005: 20).

Estas tres dimensiones: sociocultural, política y económica, se desagregan en el Reglamento del Estatuto de Autonomía. El reglamento establece que los pueblos del Caribe nicaragüense pueden elegir sus propias autoridades y crear una estructura administrativa propia (autonomía política y administrativa), regular en materia de su competencia (autonomía normativa), administrar su propio patrimonio (autonomía económica), desarrollarse bajo sus propias formas de organización (autonomía organizativa), y promover y preservar sus culturas (autonomía cultural).

Estos elementos son reconocidos generalmente por la juventud. En relación a sus percepciones sobre el régimen es importante resaltar que el $89 \%$ de encuestadas y encuestados valoró que la autonomía es importante para promover el desarrollo de la región. En la dimensión sociocultural reconocen como logros de la autonomía la existencia de las universidades regionales, el desarrollo 
de la educación intercultural y el rescate de las lenguas propias de los diferentes pueblos y grupos étnicos. En las dimensiones económicas y sociales reconocen el control que deben tener los y las costeñas sobre los recursos económicos y naturales y los planes regionales de desarrollo. Lo anterior demuestra el reconocimiento a algunos de los avances que se han obtenido en diferentes expresiones concretas de la autonomía.

Aunque reconocen avances de la autonomía, la juventud encuestada siente que el régimen autonómico no resuelve los principales problemas que enfrentan en la actualidad. Para casi el $80 \%$ de las personas en el estudio, el principal problema en la región es el desempleo y la pobreza y la mayoría siente que las expresiones administrativas de la autonomía no han logrado mejorar sus condiciones de vida.

Además el 78\% de jóvenes en la encuesta no confía en los actores políticos de los diferentes ámbitos: regional, territorial y comunal, y hay una percepción, también de la mayoría, que existe corrupción en los tres niveles. El $71 \%$ tampoco cree que las leyes sean aplicadas de forma igualitaria a toda la población. Estos datos demuestran una débil legitimidad de las estructuras legalmente constituidas ante la juventud de la región, hecho que probablemente se refleja también en la reducción de la participación en las elecciones regionales, que ha caído de $78 \%$, en 1990 , a $41 \%$ en 2010. (Gráfico 1.)

\section{Gráfico 1: Participación en Elecciones Regionales, 1990 a 2010}



Es interesante notar que a pesar de su insatisfacción con la capacidad administrativa del régimen autonómico, la mayoría planteó que no considera necesario hacer reformas a la ley de autonomía, restringir la participación a los partidos regionales, o tener elección directa del gobernador o gobernadora.

Las personas consideradas para este estudio -en edades entre dieciséis y veintinueve años- conforman la primera generación nacida y criada bajo el régimen autonómico. A diferencia de las generaciones anteriores, estos adolescentes y jóvenes no fueron directamente afectados por la lucha armada y política que culminó con el Estatuto de Autonomía, lo cual sugiere un grado menor de apropiación del proceso que condujo a la creación del régimen político actualmente en vigor, al igual que de sus particularidades y evolución.

Por otra parte es de celebrar como una probable consecuencia de las propias características del sistema político en que estos jóvenes han crecido, que el descontento que expresan hacia él no se refleja en propuestas de toma de acciones violentas, como ha sucedido en muchas ocasiones anteriores en la historia del Caribe nicaragüense, incluyendo el mismo proceso que engendró el Estatuto de Autonomía vigente (Hansell, 1999).

\section{Grado de agencia de adolescentes y jóvenes en la RACS}

Se define como agencia la formulación de metas por parte de jóvenes y de adolescentes, combinadas con la existencia de capacidades requeridas y control de recursos necesarios para perseguirlas. Un punto central planteado por el estudio es el desarrollo de agencia entre jóvenes como elemento fundamental para el funcionamiento de la autonomía. Las metas y aspiraciones tienen aspectos personales, cívicos y de identidad. Sus múltiples identidades están entrelazadas: étnicas, como parte de los diferentes pueblos indígenas o comunidades afrodescendientes; de género, como hombre o mujer; etarias, como adolescentes o jóvenes, etcétera.

Estas múltiples identidades influyen en las apreciaciones de la juventud sobre sus capacidades, oportunidades y obstáculos. En la medida en que sus aspiraciones les llevan a convertirse en sujetos políticos, la juventud puede llegar a incidir en las instituciones y agentes políticos. Estas instancias a su vez serán inducidas a crear los espacios y las condiciones para potenciar las capacidades de jóvenes y adolescentes y contribuir a la satisfacción de sus principales necesidades. (Gráfico 2.)

Fuente: IPADE, 2012: pág. 15 


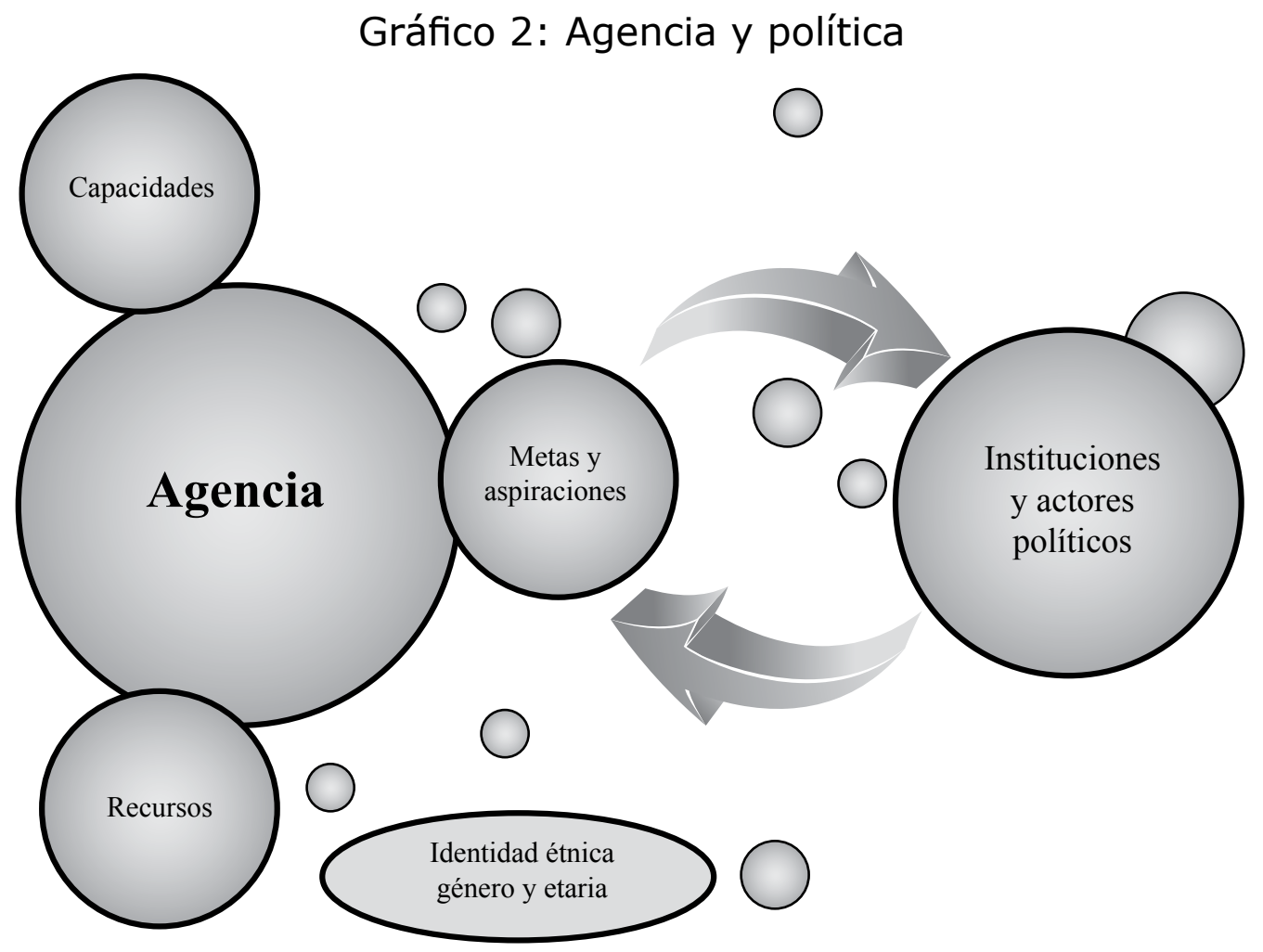

Fuente: Elaboración propia en base al marco teórico establecido en Sequeira, 2013

Para el estudio se desarrolló un índice de agencia basado en cinco variables:

1. La producción de sí mismo (la capacidad de crear oportunidades propias).

2. Funcionamiento autónomo (la capacidad de decidir lo que se quiere ser).

3. Funcionamiento autónomo reactivo (la capacidad de rechazar lo que nos perjudica).

4. La definición de metas propias.

5. La autoestima.

Basado en este índice, el 70\% de encuestados y encuestadas se encuentra "en ruta de agencia", un $22 \%$ carece de agencia, con pocas capacidades de autonomía, iniciativa, funcionamiento autónomo e incorporación de metas, y sólo el $8 \%$ posee capacidad de agencia.

\section{Agencia y participación}

La agencia está altamente ligada a la participación ciudadana. Jóvenes con capacidades y recursos necesarios deberían poder perseguir sus metas incidiendo en la esfera política. Sin embargo, en el estudio, solamente el $2.6 \%$ de las personas encuestadas tenía un índice de participación adecuado y sólo el $8.2 \%$ profesó tener un interés en la política. Aun entre jóvenes que participan activamente en organizaciones, la gran mayoría lo hace únicamente en ámbitos sociales y comunitarios, como redes juveniles y organizaciones deportivas, religiosas o estudiantiles. Además, el 59\% afirmó que no tiene preferencia partidaria. El 64\% votó en las elecciones municipales, el 58\% en las nacionales y el $57 \%$ en las regionales. La mayoría planteó que desconoce los espacios de participación.

Aunque la población de jóvenes encuestada declara que no participa por razones de tiempo, falta de interés o por no saber cómo hacerlo, también afirma que participaría si hubiera beneficios para jóvenes, comunidades y a nivel personal. Efectivamente, existen iniciativas de trabajo con jóvenes que han sido exitosas en las regiones autónomas. La movilización alrededor de las Casas Municipales de Adolescentes y Jóvenes en el marco del Programa Voz Joven en alianza con los gobiernos regionales autónomos del Caribe de Nicaragua, la Asociación de Municipios de Nicaragua (AMUNIC) y el Fondo de Población de las Naciones Unidas (UNFPA), involucró un gran número de jóvenes y adolescentes en la promoción de sus derechos reproductivos, salud sexual y reproductiva, equidad de género y nuevas visiones sobre el rol de jóvenes como sujetos plenos de derechos humanos ${ }^{1}$. En este contexto conviene destacar la alianza establecida entre EduÉxito y las Casas Municipales de Adolescentes fundadas por Voz Joven. 


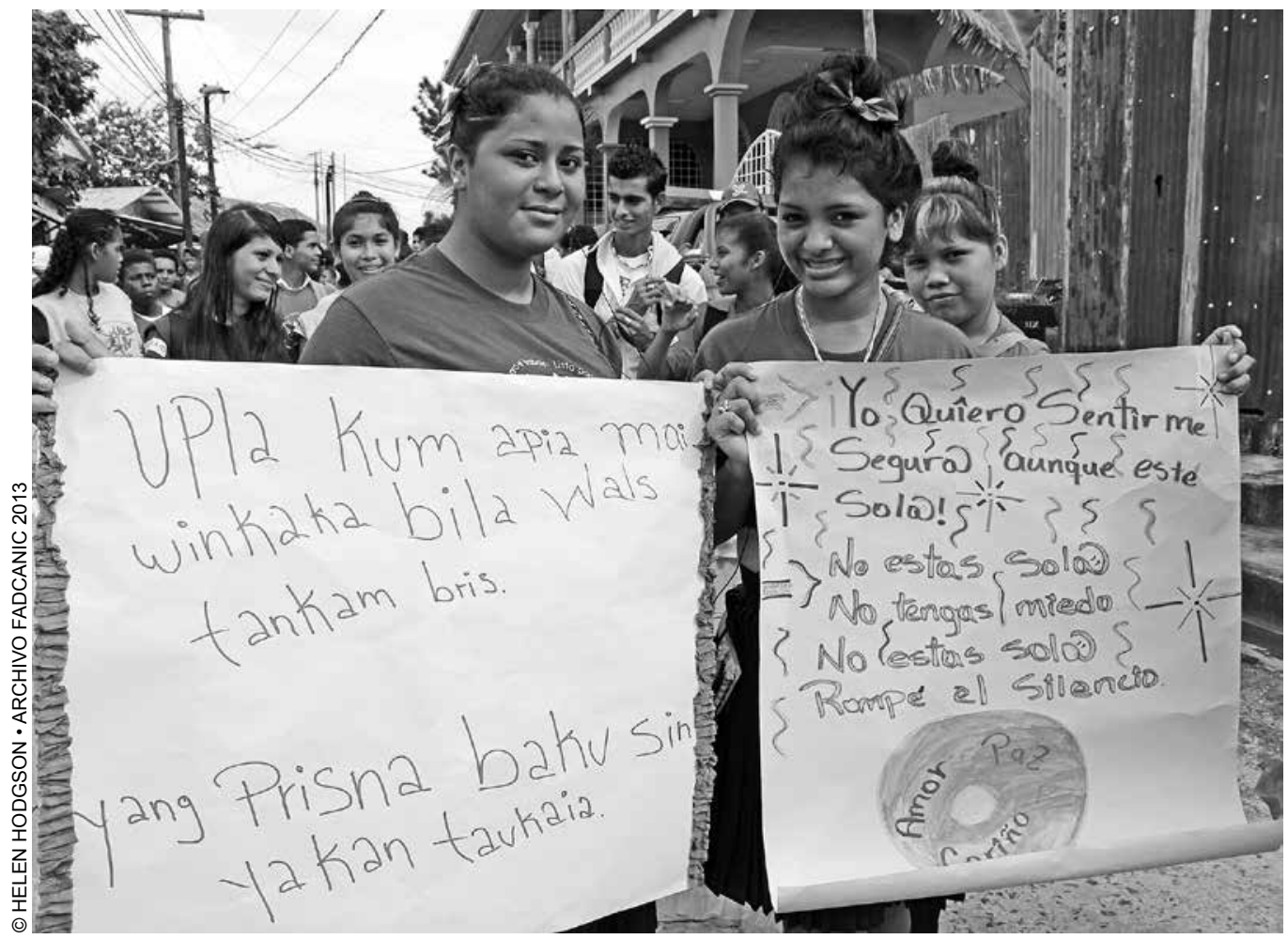

Campaña contra acoso sexual, semana de la juventud 2013.

\section{Salud sexual y reproductiva, relaciones de género y agencia}

Aunque el estudio no abarca la relación entre la salud sexual y reproductiva, las relaciones de género y el nivel de agencia, sí establecen, en la sección de Características Generales de la Muestra Encuestada, que el 40\% de jóvenes y adolescentes varones participantes en el estudio tenía hijos o hijas y en el caso de jóvenes y adolescentes mujeres, este porcentaje fue de $71 \%$. También, el Estudio sobre situación de adolescentes y jóvenes en 43 municipios de Nicaragua. Derechos, convivencia, participación y salud sexual y reproductiva, (UNFPA, 2013b), mostró que a nivel de los municipios, la edad promedio más baja de inicio de relaciones sexuales era en las regiones del Caribe nicaragüense. Por ejemplo, de la población joven y adolescentes de Bluefields se reportó una edad de inicio de relaciones sexuales de 13.5 años en varones, y de 14.7 años en mujeres ${ }^{2}$. Además, cerca de la mitad de las adolescentes y jóvenes encuestadas que habían iniciado relaciones sexuales ya había tenido al menos un embarazo (UNFPA, 2013b: 9); y en la RAAS, hasta un 73\% de las jóvenes de veinte a veinticuatro años y $37 \%$ de quince a diecinueve años, respectivamente, ya tenían al menos un hijo o hija al momento de la encuesta (UNFPA, 2013b: 32).

Esta situación es preocupante dado que el embarazo en adolescentes generalmente resulta en pérdidas de oportunidades educativas y económicas o en muertes maternas (UNFPA, 2013a). El Informe Mundial de la Población 2012 afirma que el acceso a la planificación familiar tiene efectos positivos sobre el nivel de escolarización de las mujeres jóvenes y también de sus vástagos; aumenta la participación de la mujer en la fuerza laboral; contribuye a relaciones más equitativas de poder en las parejas, y reduce la morbilidad y mortalidad (UNFPA, 2012: 84). Al mejorar la salud sexual y reproductiva (SSR), además de asegurar derechos y mejorar las vidas de mujeres y niños, se facilita su participación en la esfera política y comunitaria y se mitiga la pobreza.

Se puede encontrar más información sobre el programa Voz Joven y las Casas Municipales de Adolescentes y Jóvenes en los sitios web: https://www.vozjoven.net y http://www.unfpa.org.ni.

2 De los cinco municipios en los cuales la presente encuesta fue desarrollada, solo uno, Desembocadura de la Cruz de Ro Grande, no está incluido en el estudio arriba mencionado. Las edades promedio proporcionadas, en el estudio, para al inicio de relaciones sexuales en los otros tres municipios incluidos en nuestra encuesta son: en Corn Island 13,5 para los varones y 16 para las mujeres, en Kukra Hill 13,1 los varones y 15,2 las mujeres y en Laguna de Perlas 14,4 para los varones y 15,2 para las mujeres. 


\section{Orientaciones para programas con adolescentes y jóvenes}

En base a los resultados y análisis de este estudio se recomienda que para el abordaje de estrategias de trabajo con jóvenes y adolescentes en la RAAS, se tome en cuenta: 1) sus aspiraciones y metas, 2) las capacidades de que disponen para construir agencia y 3 ) sus actitudes hacia la participación.

Para fortalecer estos tres elementos se recomiendan en el estudio las siguientes actividades: mejorar el desempeño educativo; proveer capacidades técnicas para la inserción en el mercado laboral y promover las habilidades para la vida por medio de la educación no formal. Por otra parte se propone también patrocinar la participación juvenil en actividades y organizaciones a nivel municipal, con el objetivo de favorecer el desarrollo de habilidades de liderazgo y promover el espíritu comunitario y de grupo. Estas medidas incentivarían a los jóvenes a formular metas comunes y a encauzar sus energías en esa dirección, aumentando al mismo tiempo su interés en actuar colectivamente y promoviendo condiciones para la construcción de ciudadanía.

En resumen, el estudio recomienda un abordaje integral para fortalecer los procesos autonómicos, acercando la población juvenil a sus metas, mediante el fortalecimiento de sus capacidades y facilitando su participación en espacios institucionales y comunitarios.

\section{Reflexiones finales}

El estudio La vigencia de la autonomía en la costa Caribe Sur de Nicaragua: Encuesta de Conocimientos, Aspiraciones, Percepciones y Participación (ECAPP) de las juventudes en el régimen autonómico 2013, realizado por este mismo autor, examina la relación entre la población joven de la RAAS y el sistema político autonómico vigente en esta región.

De acuerdo al estudio, la pobreza y el desempleo en la región son los principales obstáculos que la juventud percibe para lograr sus aspiraciones de superación y la satisfacción de sus principales necesidades. Por eso es fundamental continuar los esfuerzos por reducirlos. Mientras la población joven no perciba una mejora en sus oportunidades para alcanzar el bienestar, seguirán opinando que la autonomía no resuelve sus necesidades más sentidas.

Los resultados de la investigación revelan también insatisfacción por parte de la juventud, por el grado de atención a sus principales necesidades y el descontento con el desempeño de los actores e instituciones del régimen autonómico. Paralelamente, los resultados de la encuesta indican un grado limitado de agencia y una escasa participación de la población joven en el ámbito de las cosas públicas.

Es indispensable, tanto para el futuro de la región como para el desarrollo personal de la juventud del Caribe nicaragüense, que esta población joven mejore su capacidad de tomar su propio destino en sus manos, a través de la adquisición de competencias, control de recursos y definición de metas. Tal desarrollo les conducirá también necesariamente a incidir en el control del sistema político del régimen autónomo.

Por su parte, el régimen tiene que facilitar el alcance de las aspiraciones de la población joven y fortalecer sus competencias. Para obtener ese objetivo es necesario tomar medidas que desarrollen sus conocimientos sobre el régimen autonómico, les acerquen de los actores políticos y les inciten a participar y a apropiarse de las instituciones que inciden sobre ellos.

Desde esa perspectiva es vital que se creen espacios regionales, territoriales, municipales y comunales, que permitan y promuevan la participación de jóvenes en los ámbitos sociales y culturales, pero también políticos, de la autonomía. Esto ayudaría a mejorar su percepción de las instituciones y actores autonómicos, fortaleciendo al mismo tiempo la legitimidad de los aparatos administrativos a los niveles regionales, municipales, territoriales y comunales.

Dado el carácter multiétnico y multicultural de la sociedad costeña, los espacios y mecanismos de participación deben tomar en cuenta y potencializar el diálogo intercultural. Por otra parte, aunque el estudio no lo mencione directamente, también es necesario poner atención a los factores que limitan la participación activa de las mujeres jóvenes, como las relaciones desiguales de género y la falta de acceso a los servicios de salud sexual y reproductiva. Estos limitan su participación y su agencia, y reproducen el ciclo de la pobreza, debilitando la autonomía.

Finalmente es pertinente señalar, en esta discusión, que el bono demográfico, producido por las todavía altas tasas de fecundidad y un descenso en la mortalidad aún moderado, está produciendo actualmente una creciente proporción de población en edad de trabajar, reduciendo así el peso relativo de la población dependiente. Esta configuración demográfica podría ser utilizada para producir un salto económico en la región. Para lograr ese objetivo se requiere de una planificación e inversión estratégicamente adecuada, que asegure que la juventud tenga las capacidades y encuentren las oportunidades para trabajar y producir: ésa es también, precisamente, la mayor aspiración de la juventud que participó en la encuesta. 


\section{BIBLIOGRAFÍA}

Centro de Derechos Humanos, Ciudadanos y Autonómicos (CEDEHCA) Agenda de Jóvenes Afrodescendientes. Bluefields: CEDEHCA.

Consejo Regional Autónomo del Atlántico Sur. 2010. Agenda Regional de Adolescentes y Jóvenes de la Región Autónomo del Atlántico Sur. Bluefields: UNFPA/ Consejo Regional Autónomo del Atlántico Sur.

Delgadillo, Maritza. 2010. El bono demográfico y sus efectos en el desarrollo económico y social de Nicaragua. UNFPA, Nicaragua; CEPAL/CELADE, 2010

Fondo de Población de las Naciones Unidas (UNFPA). 2013a. Estado de la población mundial 2013: Enfrentar el reto del embarazo en adolescentes Maternidad en la niñez. New York: UNFPA.

Fondo de Población de las Naciones Unidas (UNFPA). 2013b

Fondo de Población de las Naciones Unidas (UNFPA). 2012. Estado de la población mundial 2012: Sí a la Opción, No al Azar. Planificación de la Familia, Derechos Humanos y Desarrollo. New York: UNFPA.

Fondo de Población de las Naciones Unidas (UNFPA). 2007. Marco para la Acción con Adolescentes y Jóvenes, UNFPA.

Fondo para el Desarrollo de los Pueblos Indígenas de América Latina y el Caribe. "Monitoreo de derechos y buen vivir de los pueblos indígenas: una contribución del Fondo Indígena para el trabajo regional" en Stankovitch, Mara. (Ed.). 2008. Indicadores Relevantes para los Pueblos Indigenas: Un texto de Referencia. Centro Internacional para Estudios de Políticas y Educación de Pueblos Indígenas. Tebtebba Foundation. Baguio City, Filipinas.

Hansell, Timothy. 1999. "Saga of the Yatama" Americas Update, Toronto: Latin American Working Group (LAWG); Jesuit Centre for Social Faith and Justice; Canada Latin America Resource Centre (CLARC).

Instituto para el Desarrollo y la Democracia. 2012. Catálogo Estadístico de Elecciones en Nicaragua 1990 - 2011. 2 da ed. Managua: IPADE.

Instituto Nacional de Información de Desarrollo. 2007. Estimaciones y Proyecciones de Población Nacional, Departamental y Municipal (Revisión 2007). Managua: INIDE.

Naciones Unidas. 1966. Pacto International de Derechos Económicos, Sociales y Culturales. Comité de Derechos Económicos, Sociales y Culturales de las Naciones Unidas.

Naciones Unidas. 1966. Pacto Internacional de Derechos Civiles y Políticos de las Naciones Unidas. Comité de Derechos Humanos de las Naciones Unidas.

Naciones Unidas. 2007. Declaración de las Naciones Unidas sobre los derechos de los pueblos indígenas.

Nicaragua. 2003. "Ley 445: Ley del Régimen de Propiedad Comunal de los Pueblos indígenas y Comunidades étnicas de las Regiones Autónomas de la Costa Atlántica de Nicaragua y de los Ríos Bocay, Coco, Indio y Maíz." La Gaceta Diario Oficial 16(445).

Nicaragua. 2003. "Reglamento a la Ley 28 Estatuto de Autonomía de las dos Regiones de la Costa Atlántica de Nicaragua." La Gaceta Diario Oficial: 16(186).

Nicaragua. 1987. "Ley 28 Estatuto de Autonomía de las dos Regiones de la Costa Atlántica de Nicaragua." La Gaceta Diario Oficial: 238.

Programa de las Naciones Unidas para el Desarrollo. 2011. Informe Nacional Sobre Desarrollo Humano 2011. Las juventudes construyendo Nicaragua. Nicaragua: PNUD.

Programa de las Naciones Unidas para el Desarrollo. 2005. Informe de Desarrollo Humano 2005. Las Regiones Autónomas de la Costa Caribe ¿Nicaragua asume su diversidad? Nicaragua: PNUD.

Sequeira Rankin, Francisco. 2013. La vigencia de la autonomía en la costa Caribe Sur de Nicaragua: Encuesta de Conocimientos, Aspiraciones, Percepciones y Formas de Participación (ECAPP) de las Juventudes en el Régimen Autonómico 2013. Nicaragua: FADCANIC. 